\title{
Laser-induced breakdown spectroscopy: Extending its application to soil $\mathrm{pH}$ measurements
}

\author{
Edilene Cristina Ferreira ${ }^{\mathrm{a}, *}$, José A. Gomes Neto ${ }^{a}$, Débora M.B.P. Milori ${ }^{\mathrm{b}}$, \\ Ednaldo José Ferreira ${ }^{b}$, Jesús Manuel Anzano ${ }^{c}$ \\ a São Paulo State University - UNESP, Analytical Chemistry Department, Rua Prof. Francisco Degni 55, CEP 14800-060, Araraquara, SP, Brazil \\ ${ }^{\mathrm{b}}$ Embrapa Agricultural Instrumentation, Rua XV de Novembro 1452, CEP 13560-970, São Carlos, SP, Brazil \\ ' Laser Laboratory E' Environment, Faculty of Sciences, University of Zaragoza, C/. Pedro Cerbuna 12, 50009, Zaragoza, Spain
}

\section{A R T I C L E I N F O}

\section{Article history:}

Received 17 October 2014

Accepted 8 June 2015

Available online 14 June 2015

\section{Keywords:}

Soil pH determination

PLS

LIBS

\begin{abstract}
A B S T R A C T
Acid-base equilibria are involved in almost all the processes that occur in soil. The bioavailability of nutrients for plants, for instance, depends on the solubilization of mineral nutrients in the soil solution, which is a $\mathrm{pH}$-dependent process. The determination of $\mathrm{pH}$ in soil solutions is usually carried out by potentiometry using a glass membrane electrode, after extracting some of the soil components with water or $\mathrm{CaCl}_{2}$ solution. The present work describes a simple method for determining the $\mathrm{pH}$ of soil, using laser-induced breakdown spectroscopy (LIBS). Sixty samples presenting different textural composition and $\mathrm{pH}$ (previously determined by potentiometry) were employed. The samples were divided into a calibration set with fifty samples and a validation set with ten samples. LIBS spectra were recorded for each pelleted sample using laser pulse energy of $115 \mathrm{~mJ}$. The intensities of thirty-two emission lines for $\mathrm{Al}, \mathrm{Ca}, \mathrm{H}$, and $\mathrm{O}$ were used to fit a partial least squares (PLS) model. The model was validated by prediction of the $\mathrm{pH}$ of the validation set samples, which showed good agreement with the reference values. The prediction mean absolute error was $0.3 \mathrm{pH}$ units and the root mean square error of the prediction was 0.4 . These results highlight the potential of LIBS for use in other applications beyond elemental composition determinations. For soil analysis, the proposed method offers the possibility of determining $\mathrm{pH}$, in addition to nutrients and contaminants, using a single LIBS measurement.
\end{abstract}

(c) 2015 Elsevier B.V. All rights reserved.

\section{Introduction}

Acid-base equilibria are involved in almost all soil processes, and the physical, chemical, and biological properties of soil are all influenced by $\mathrm{pH}$ [1]. The bioavailability of plant nutrients depends on the solubilization of minerals or other nutrients in the soil solution, and this process is $\mathrm{pH}$ dependent. The $\mathrm{pH}$ can also affect the activity of microorganisms, altering the rate of decomposition of soil organic matter and consequently the release of nutrients [2]. The determination of soil $\mathrm{pH}$ is therefore crucial for agricultural soil use. For example, the correction of soil $\mathrm{pH}$ (in the case of soils presenting high acidity) by the application of bases relies on $\mathrm{pH}$ measurements.

The $\mathrm{pH}$ of mineral soils is normally determined using slurries of water and soil [1]. The use of $\mathrm{CaCl}_{2}$ solution for determining the $\mathrm{pH}$ of soils was proposed by Schofield and Taylor [3], with the aim of minimizing interferences due to particles suspended in the soil solution and variable salt contents.

\footnotetext{
* Corresponding author. Tel.: + 551633019616 .

E-mail address: edilene@iq.unesp.br (E.C. Ferreira).
}

In 1990, the Soil Science Society of America (SSSA) and the Association of Official Agricultural Chemists (AOAC) decided to conduct collaborative studies in order to validate techniques applied to soils. Four potentiometric methods for soil $\mathrm{pH}$ measurement were considered, according to the characteristics of the soil: (a) pH measurement in a soil suspension obtained by stirring the sample with distilled or deionized water, applied to mineral soil samples containing less than $17 \%$ of organic carbon; (b) pH measurement in a soil suspension obtained by stirring the sample with $0.01 \mathrm{~mol} \mathrm{~L}^{-1} \mathrm{CaCl}_{2}$ solution, applied to mineral soils with variable salt content; (c) $\mathrm{pH}$ measurement in a soil paste obtained by moistening the sample with distilled or deionized water, applied to soils containing large amounts of soluble salts and sufficient exchangeable sodium; (d) pH measurement in an aqueous soil extract, applied to soils containing organic carbon at levels equal to or greater than $17 \%$. Based on the results of this study, methods for $\mathrm{pH}$ measurements of mineral, saline-sodic, and organic soils were adopted by AOAC International [4].

Although the methods recommended by AOAC are effective, they involve the use of potentiometric measurements, which require the preparation of a soil solution for each sample [5]. Moreover, the dependence of the sample preparation method on the characteristics of the soil 
requires previous soil characterization, which complicates the analysis of a heterogeneous set of soil samples.

The present work proposes a new and simple method for the determination of soil $\mathrm{pH}$, based on laser-induced breakdown spectroscopy (LIBS). LIBS is an optical technique capable of performing direct multielement analysis, eliminating the need for the conventional preparation of sample solutions before analysis [6,7]. Moreover, LIBS has potential for use in analyses conducted in situ. LIBS measurements are based on recording light emissions from atomic and ionic species present in a sample, after their excitation in a transient plasma produced by a laser pulse. Typically, a LIBS spectrum provides qualitative information about the elemental composition of the sample, although the signal intensities for each wavelength can be used for quantitative determinations $[7,8]$. In this proposal, the LIBS emission lines of elements related to soil acidity were selected to fit a multivariate model for soil $\mathrm{pH}$ determination.

\section{Experimental}

A set of sixty soil samples with distinct textures was provided by the Agronomic Institute of Campinas (AIC, Campinas, Brazil). The samples were dried at $40{ }^{\circ} \mathrm{C}$ in an oven with air circulation and then passed through a sieve with $2 \mathrm{~mm}$ mesh openings.

The set of 60 samples was divided into two subsets: calibration and validation sets, containing 50 and 10 samples, respectively. The samples allocated to each group were randomly selected. No heuristics were used to balance the number of the samples in the calibration and validation sets, due to the relatively small number of samples.

As part of an AIC proficiency-testing program, several parameters (including $\mathrm{pH}$ ) were determined for each soil sample by 89 Brazilian laboratories. The average $\mathrm{pH}$ values reported by AIC were used as reference values in the present work. The pH values of the 60 samples were from 4.0 to 6.3 , with standard deviations ranging from 0.1 to $0.2 \mathrm{pH}$ units.

For the LIBS analysis, the samples were submitted to cryogenic grinding in order to reduce their heterogeneity, using a SpexCertiprep Model 6750 cryogenic mill operated at liquid nitrogen temperature $\left(-196{ }^{\circ} \mathrm{C}\right)$. The grinding program consisted of two grinding steps of $2 \mathrm{~min}, 3 \mathrm{~min}$ for pre-cooling, and $2 \mathrm{~min}$ for cooling between the grinding steps. This procedure produced particles in the 60-120 $\mu \mathrm{m}$ size range. The ground samples were used to prepare pellets by applying 10-ton pressure on a portion $(\sim 500 \mathrm{mg})$ of sample during $3 \mathrm{~min}$. The pellets were analyzed by LIBS, recording ten spectra for each sample. The spectra obtained for each sample were then averaged, without any preprocessing, to obtain a representative spectrum per sample.

The LIBS system comprised a Nd:YAG laser (Brilliant Quantel, Q-Switched) with a second harmonic generator (SHG), an xyz stage carrying the sample (Standa 011957), a spectrograph, and an intensified charge-coupled device (ICCD) detector. The characteristics of the laser were maximum laser pulse energy of $115 \mathrm{~mJ}$ (operating at $532 \mathrm{~nm}$ ), $4.4 \mathrm{~ns}$ pulse duration, and $10 \mathrm{~Hz}$ repetition frequency. The plasma light was collected and transported to the spectrograph through a lens and an optical fiber. An external collimator was used as the collecting lens. The position of the fiber relative to the collecting lens was adjusted by means of a divergent beam from a diode laser (HE-OPI-0009, Andor) connected to the fiber, which was focused on the sample position by the collecting system. This arrangement ensured that the light emitted by the sample was focused on the fiber. An Echelle spectrograph (Andor Mechelle ME5000, focal length 195 mm, F/7, spectral range from 200 to $975 \mathrm{~nm}$ ) was coupled to an ICCD detector (Andor iStar DH734, $1024 \times 1024$ pixels, $13.6 \times 13.6 \mu^{2} /$ pixel, $18 \mathrm{~mm}$ intensifier diameter). This system was calibrated using a mercury-argon lamp (Ocean Optics HG-1, 253-922 nm).

The LIBS experimental conditions established for acquisition of the spectra were $115 \mathrm{~mJ}$ laser pulse energy, two pulses per site, delay time of $400 \mathrm{~ns}$, and integration time gate of $25 \mu \mathrm{s}$.
Wavelengths corresponding to the core lines of $\mathrm{Al}, \mathrm{Ca}, \mathrm{H}$, and $\mathrm{O}$, and wavelengths in the wings of these core lines, were selected as follows: $373.665 \mathrm{~nm}$ (wing of the Ca II line), $373.688 \mathrm{~nm}$ (Ca II line), $393.308 \mathrm{~nm}$ (wing of the Ca II line), $393.333 \mathrm{~nm}$ (Ca II line), $393.357 \mathrm{~nm}$ (wing of the Ca II line), $394.419 \mathrm{~nm}$ (Al I line), $396.103 \mathrm{~nm}$ (wing of the Al I line), $396.128 \mathrm{~nm}$ (wing of the Al I line), $396.153 \mathrm{~nm}$ (Al I line), $396.178 \mathrm{~nm}$ (wing of the Al I line), $396.799 \mathrm{~nm}$ (wing of the Ca II line), $396.824 \mathrm{~nm}$ (Ca II line), $655.461 \mathrm{~nm}$ (O II line), $655.502 \mathrm{~nm}$ (wing of the O II line), 655.542 (wing of the O II line), 655.582 (wing of the $\mathrm{O}$ II line), $655.622 \mathrm{~nm}$ (wing of the O II line), $655.662 \mathrm{~nm}$ (wing of the O II line), $656.065 \mathrm{~nm}$ (wing of the H I line), $656.105 \mathrm{~nm}$ (H I line), $656.145 \mathrm{~nm}$ (wing of the $\mathrm{H} I$ line), $656.306 \mathrm{~nm}$ (wing of the $\mathrm{HI}$ line), $656.346 \mathrm{~nm}$ (wing of the $\mathrm{HI}$ line), $656.387 \mathrm{~nm}$ (wing of the $\mathrm{H}$ I line), $656.427 \mathrm{~nm}$ (wing of the $\mathrm{H} \mathrm{I}$ line), $656.467 \mathrm{~nm}$ (wing of the $\mathrm{O}$ II line), $656.507 \mathrm{~nm}$ (O II line), 656.991 (wing of the $\mathrm{O}$ II line), $657.031 \mathrm{~nm}$ (wing of the $O$ II line), $657.071 \mathrm{~nm}$ (wing of the $O$ II line), $657.112 \mathrm{~nm}$ (O II line), and $657.152 \mathrm{~nm}$ (wing of the O II line). Using the selected lines, a partial least squares (PLS) model for the prediction of soil $\mathrm{pH}$ was constructed using centralization of the data on the average, with five latent variables. The calibration model took account of at least nine sources of variability, considering only the centers of the selected lines. The Weka v. 3.5.7 system (Waikato Environment for Knowledge Analysis) was used to build and validate the calibration model [9].

\section{Results and discussion}

A heterogeneous set of soils (in terms of texture) was used in order to obtain a robust model for $\mathrm{pH}$ determination, independent of the sample matrix. The proposed model could therefore offer important advantages compared to the AOAC method, where different textural classes of soils require separate extraction procedures [4].

The influence of $\mathrm{pH}$ on processes occurring in the soil is complex. Some well-known processes in which the $\mathrm{pH}$ varies as a function of exchangeable cations were therefore used as criteria to select independent variables for the calibration model. Soil liming, a procedure employed to increase soil $\mathrm{pH}$, is performed by adding limestone derived from calcareous sedimentary rock containing calcium carbonate at levels exceeding $30 \%$ [10]. The presence of calcium in the soil can therefore be directly correlated to the $\mathrm{pH}$. At acid $\mathrm{pH}$, exchangeable aluminum predominates, while with liming, $\mathrm{Al}^{+}$is progressively precipitated and remains in the form of $\mathrm{Al}(\mathrm{OH})_{3}$ in alkaline soil [11]. Considering these processes, wavelengths corresponding to the emission lines of $\mathrm{Ca}, \mathrm{Al}, \mathrm{H}$, and $\mathrm{O}$ were selected. Additionally, with the aim of circumventing matrix effects and self-absorption, which could affect the cores of the selected emission lines, several wavelengths in the wings of the peaks were also selected. It is important to mention that although organic acids influence soil $\mathrm{pH}$, the carbon line at $247.856 \mathrm{~nm}$ showed an unresolved iron interference ( $247.857 \mathrm{~nm})$. Since the samples presented high iron contents, this carbon line could not be included. The spectral regions of the selected lines are shown in Fig. 1. The labeled peaks correspond to Ca II (373.688 nm) (Fig. 1a); Ca II (393.333 nm), Al I (394.419 nm), Al I (396.153 nm), and Ca II (396.824 nm) (Fig. 1b); and O II (655.461 nm), H I (656.105 nm), O II (656.507 nm), and O II (657.112 nm) (Fig. 1c).

An average spectrum was calculated from the ten replicates measured for each sample. The coefficients of variation observed for the selected lines ranged from $8 \%$ to $30 \%$, depending on the sample composition and the wavelength used. Bousquet et al. [12] reported variability ranging from $10 \%$ to $25 \%$ in LIBS signals resulting from the ablation of a typical soil prepared as a pressed pellet. On this basis, the present data were considered acceptable for the construction of a multivariate calibration model.

To build the calibration model, the emission intensities at each selected wavelength for each calibration sample (independent variables) were organized in line vectors to obtain the X matrix $(50 \times 32)$, while $\mathrm{pH}$ values (dependent variables) corresponding to each calibration 


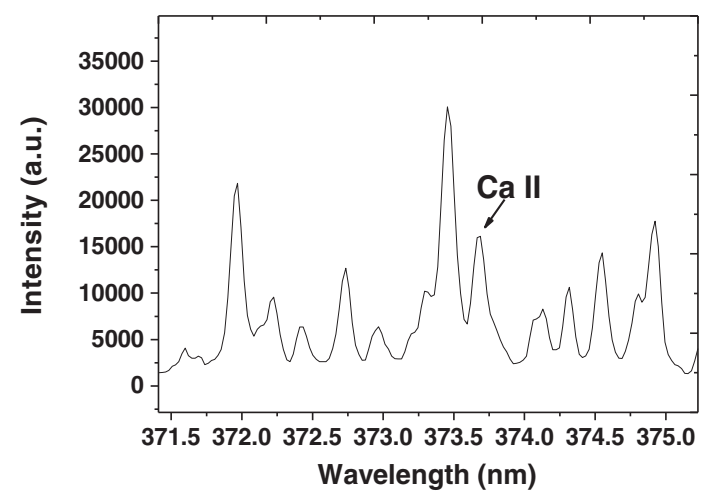

(a)

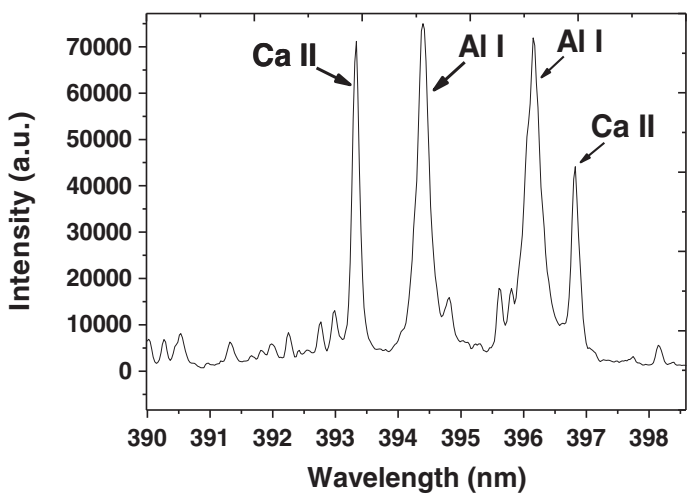

(b)

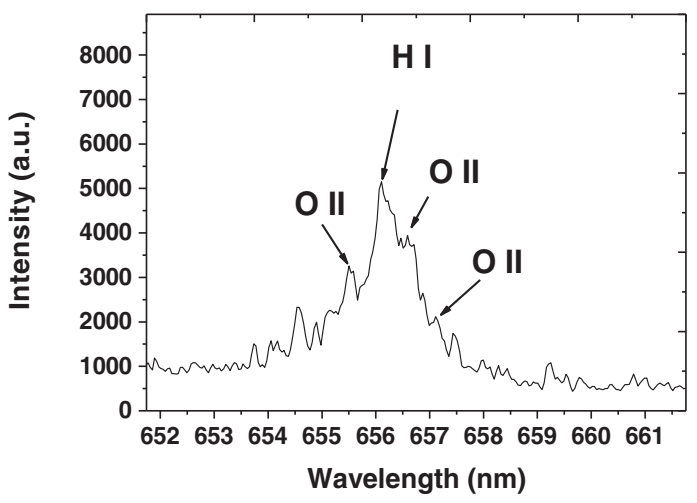

(c)

Fig. 1. Extracts of a typical LIBS spectrum of a soil sample, highlighting the cores of selected emission lines: (a) Ca II 373.688 nm, (b) Ca II 393.333 nm, Al I 394.419 nm, Al I 396.153 nm, Ca II 396.824 nm, (c) O II 655.461 nm, H I 656.105 nm, O II 656.507 nm, and O II $657.112 \mathrm{~nm}$.

sample were organized in a column vector to obtain the Y matrix $(50 \times 1)$. From these matrices, a partial least squares (PLS) model was built and optimized with respect to the numbers of latent variables. The optimization results indicated that the use of five latent variables provided the best model fitting $(\mathrm{R}=0.8661)$.

After obtaining the calibration model, it was applied for prediction of the $\mathrm{pH}$ of the 10 validation set samples. The results of the method validation are shown in Table 1 . The values predicted by the proposed model showed a positive correlation with the reference $\mathrm{pH}$ values, with a root mean square error of prediction (RMSEP) of 0.4. The mean absolute error (MAE) was $0.3 \mathrm{pH}$ units, which can be considered acceptable for this type of determination. Furthermore, the sample with pH 6.2 showed the largest absolute error of prediction $(\mathrm{AE}=1.3)$, which acted to increase the MAE. It is important to mention that this same sample showed the largest relative standard deviation calculated from determinations using the reference method ( $R S D=3.2 \%, n=89$ ). These results suggest that the physical characteristics of this sample interfered with the $\mathrm{pH}$ measurements made using both methods.
Table 1

Reference and predicted $\mathrm{pH}$ values, and absolute errors of prediction, for ten samples of the validation set.

\begin{tabular}{lll}
\hline pH reference value & $\mathrm{pH}$ predicted value & Absolute error ${ }^{*}$ \\
\hline 6.2 & 4.9 & 1.3 \\
5.4 & 5.0 & 0.6 \\
4.5 & 4.4 & 0.1 \\
5.4 & 5.3 & 0.1 \\
5.0 & 5.1 & 0.1 \\
4.5 & 4.5 & 0.0 \\
5.6 & 5.6 & 0.0 \\
4.1 & 4.4 & 0.3 \\
4.8 & 4.7 & 0.1 \\
5.9 & 5.7 & 0.2 \\
\hline$*$ Mean absolute error (MAE) $=0.3$ &
\end{tabular}

The variances observed for the dependent variable $(\mathrm{pH})$, of around $6 \%$, were lower compared to those for the independent variables (wavelengths), which ranged from $8 \%$ to $30 \%$. This indicates that despite the difficulties that high signal variability can cause for quantitative analysis of soil by LIBS, calibration models that use multiple variables (such as PLS) can minimize the influence of the variances as well as matrix and self-absorption effects.

These findings highlight the potential of LIBS for obtaining other important sample information, in addition to elemental composition, demonstrating that the technique can make a significant contribution to soil science.

\section{Conclusions}

A clean and simple method for the determination of soil pH by LIBS is proposed, using a PLS calibration model and taking into account variables corresponding to elements that influence soil $\mathrm{pH}$. The $\mathrm{pH}$ values obtained by the LIBS and reference methods were positively correlated, with low MAE ( $0.3 \mathrm{pH}$ units). The results show the potential of LIBS for evaluating soil acidity, with advantages over traditional potentiometric methods including low analytical cost, no chemical waste generation, and a single analytical procedure irrespective of the soil composition. Furthermore, the proposed method offers the possibility of determining $\mathrm{pH}$, as well as nutrients and contaminants, in a single LIBS measurement.

Future studies should be carried out to obtain a better understanding of the effects of the selected variables and their contributions to the performance of the calibration model.

\section{Acknowledgements}

The authors thank the São Paulo State Research Foundation (FAPESP, grants 2006/61741-4 and 2014/26185-0), Fundación Carolina, the European Social Fund, the Government of Aragón, and the University of Zaragoza (proposal \#E75).

\section{References}

[1] E.O. McLean, Soil pH and Lime Requirement, in: A.L. Page, R.H. Miller, D.R. Keeney (Eds.), Methods of Soil Analysis. Part 2. Chemical and Microbiological Properties, American Society of Agronomy, Soil Science Society of America, Madison 1982, pp. 199-224.

[2] J.B. Jones Jr., Plant Nutrition and Soil Fertility Manual, 2nd ed. CRC Press, Taylor \& Francis Group, Boca Raton, 2012.

[3] R.K. Schofield, A.W. Taylor, The measurement of soil pH, Soil Sci. Soc. Am. Proc. 19 (1955) 164-167.

[4] Y.P. Kalra, Determination of $\mathrm{pH}$ of soils by different methods: collaborative study, J. AOAC Int. 78 (1995) 310-324.

[5] D.A. Skoog, D.M. West, F.J. Holler, Fundamentals of Analytical Chemistry, 6th ed. Saunders College Publishing, New York, 1992.

[6] A.W. Miziolek, V. Palleschi, I. Schechter, Laser Induced Breakdown Spectroscopy-Fundamentals and Applications, Cambridge University Press, Cambridge, 2012. 
[7] D.W. Hahn, N. Omenetto, Laser-induced breakdown spectroscopy (LIBS). Part II: Review of instrumental and methodological approaches to material analysis and applications to different fields, Appl. Spectrosc. 66 (2012) 347-419.

[8] D.A. Cremers, L.J. Radziemski, Handbook of Laser-Induced Breakdown Spectroscopy, Wiley \& Sons, Chichester, 2006.

[9] M. Hall, E. Frank, G. Holmes, B. Pfahringer, P. Reutemann, I.H. Witten, The WEKA data mining software: an update, SIGKDD Explor. 11 (2009) 10-17.

[10] S.L. Tisdale, W.L. Nelson, Soil Fertility and Fertilizers, 2nd ed. Macmillan, New York 1966.
[11] N.T. Coleman, D. Craig, The spontaneous alteration of hydrogen clay, Soil Sci. 91 (1961) 14-18.

[12] B. Bousquet, G. Travaillé, A. Ismaël, L. Canioni, K. Michel-Le Pierrès, E. Brasseur, S. Roy, I. le Hecho, M. Larregieu, S. Tellier, M. Potin-Gautier, T. Boriachon, P. Wazen, A. Diard, S. Belbèze, Development of a mobile system based on laser-induced breakdown spectroscopy and dedicated to in situ analysis of polluted soils, Spectrochim. Acta Part B 63 (2008) 1085-1090. 